\title{
Eccoci al numero 100 di Sinergie
}

Marzo 1983: dopo lunghe discussioni sul senso, la linea, il titolo di una rivista che accreditasse i lavori del Consorzio Universitario di Economia Industriale e Manageriale (CUEIM), nasce Sinergie.

Agosto 2016: esce il numero 100 della rivista.

Trentatré anni di attività, un periodo che proietta la rivista negli orizzonti della longevità tra i journal che si occupano di management e governo delle organizzazioni siano esse private o pubbliche.

E un traguardo che è arrivato quasi inaspettato, protési in quella continua azione redazionale che con il lavoro di tanti ci ha condotto, quasi increduli, sin qui.

Impensabile proporre in breve una lettura della varietà di passaggi che hanno contraddistinto il divenire della rivista, quasi occorrerebbe un progetto di ricerca per farlo.

975 autori, 146 referee, 48.031 visite al sito nell'ultimo anno, oltre 16.000 paper scaricati dal 2013. Questi solo alcuni numeri della rivista che potremmo dire ci è cresciuta sotto gli occhi con il lavoro spesso silenzioso di coloro che hanno creduto nella sua proposta editoriale.

E allora cosa dire in apertura di questo n. 100? A lungo abbiamo pensato a questo perché si tratta di scelta difficile in quanto connessa anche ad aspetti di tipo emozionale ed affettivo che vanno ben al di là del lavoro e dell'oggettività dei fatti.

Ma poi, convinti che "spesso il futuro stia alle nostre spalle", se vi si presta la necessaria attenzione in una prospettiva rivolta all'avvenire, abbiamo immaginato un ritorno alle origini.

Così, anche ritenendo che tanti Lettori, soprattutto i più giovani, non abbiano avuto la ventura di leggere l'Editoriale del numero "zero" del Fondatore della rivista Giovanni Panati, abbiamo pensato di riprenderlo in alcune sue parti, ad iniziare da quella relativa alla scelta del titolo.

“.....Sinergia è termine di origine greca (syn, insieme ed ergon, lavoro), introvabile nel classico dizionario della lingua italiana dei Tommaseo. $\dot{E}$ entrato di recente nei linguaggio moderno, soprattutto ad opera della scienza medica, dove lo si usa per indicare l'effetto provocato sull'organismo dall'attività simultanea di vari organi o funzioni, oppure per indicare l'effetto esaltante delle proprietà e dell'attività di un farmaco quando venga somministrato congiuntamente ad un altro di proprietà analoghe o complementari. Ma anche in economia lo si usa ormai correntemente per indicare uniazione combinata capace di produrre (generalmente, ma non necessariamente) un risultato maggiore della mera sommatoria delle singole forze o componenti costituenti l'azione stessa. In breve, esso indica un'esaltazione delle caratteristiche positive (o negative, se il segno risultante fosse tale) di azioni, forze o componenti operanti insieme.

Chiamando Sinergie questa rivista abbiamo voluto perciò sottolineare la speranza e l'augurio che il concorso simultaneo di persone appartenenti a discipline diverse, con orientamenti e capacità differenti (caso assai frequente 
sinergie Vol. 34, N. 100, 2016

nella maggior parte delle ricerche tecnoeconomiche), abbia a sortire un effetto superiore ai "valore" dei singoli contributi.

Pascoli ed Einaudi (ma prima di loro Montaigne e Pascal) direbbero che il miele è un risultato sinergetico del "lavoro" delle api e dei fiori. L'immagine può essere fuorviante e non conviene insistere....."

E dal titolo breve è il passaggio al senso del progetto originale della rivista.

“... Progettare e realizzare una nuova rivista (che, per quanto modesta, costringe ad uniattività pubblica) è fatica grande, per sobbarcarsi la quale occorre non poco entusiasmo ed ottimismo, se è vero che l'ottimismo è una capacità personale di investire le proprie energie e i propri interessi. L'intensità di ottimismo necessaria dipende in realtà dal giudizio sullambiente socioculturale: ne occorre una dose eroica se ci sentiamo circondati dalla crisi dei valori e della ragione, se ci vediamo sommersi dallimbarbarimento, dal deterioramento e dal non-senso. Ne basta invece una dose assai minore (ma non trascurabile) se nella nostra rappresentazione della società prevale la fiducia nell' "homo faber" e nella sua potenziale creatività pacificatrice. Lasciamo perciò al lettore di valutare il nostro sforzo.

Non bastano però entusiasmo e ottimismo. Occorre anche un minimo di volontà collaborativa e di convincimenti comuni: occorre quanto meno un comune denominatore di fiducia verso il contributo essenziale della conoscenza al processo di scoperta delle verità (scientifiche) attraverso le quali l'uomo può crescere nelle libertà.

Già nell'iter di preparazione della rivista ci è stato chiesto a quale scopo accrescere conoscenza e verità. La risposta può sembrare ingenua, ma imprescindibile: esso sta nel presupposto implicito che una maggior dose di conoscenzaverità (unita alla necessaria consapevolezza etico-sociale) consenta di migliorare nel tempo la qualità della vita.

Ma gli interrogativi rimbalzano: chi decide prevalentemente la qualità della vita del mondo in cui viviamo, cioè del mondo industrializzato? Il complesso gioco dei rapporti internazionali - che ritagliano spazi e gradi di libertà - o l'intreccio invisibile di tutti i micro e macro-interessi di uomini e gruppi, o la grande impresa, come si rispondeva in passato?

Qualunque sia la risposta ci sembra che, in ogni caso, una maggior conoscenza scientifica dei processi che conducono alle scelte economiche, tecnologiche, produttive, sociali dovrebbe offrire la possibilità di un maggior controllo sociale, la cui intensità e modalità di attuazione dipende dalletica della volontà.

Questa è la minima Weltanschauung che dà significato e guida alliniziativa del nuovo periodico...."

Ed ecco dal senso l'affacciarsi degli obiettivi originari del periodico.

“... Se obiettivo prossimo della rivista è di dare una voce al Consorzio universitario di ricerca - e a quanti vi collaborano - verso $i$ suoi associati e verso lesterno, non meno degni di considerazione sono gli obiettivi remoti: si è voluto creare una potenzialità agglomerativa, un punto d'incontro e di 
dibattito, una palestra aperta a giovani studiosi, ricercatori e intellettuali d'impresa, con i quali il rapporto può apparentemente risolversi in un momento di "costrizione" alla riflessione (scrivere è sempre più "costrittivo" che parlare, e perciò più fecondo, perché si è appunto costretti a chiarire a se stessi le idee che si vogliono comunicare)....."

E trentatré anni dopo e cento fascicoli dopo eccoci qui. Siamo giunti al numero 100 con tanti cambiamenti che hanno portato la rivista a rappresentare in qualche modo la storia degli studiosi di Economia e gestione delle imprese, anche attraverso il Convegno Annuale ora alla sua ventinovesima edizione curata in collaborazione con Sima.

Quale sia la qualità del risultato cui siamo pervenuti non tocca certo noi dire. Tocca, invece, a noi ringraziare di cuore e ad alta voce tutti i nostri Lettori che hanno percorso le pagine della rivista rendendola vitale, gli Autori - professori, giovani ricercatori e dottorandi - che con il loro contributo di riflessione hanno creato l'interesse intorno al periodico, i Referee che hanno assicurato il valore delle proposte, i Colleghi del Comitato Scientifico e gli Associate Editors che hanno contribuito a definirne la direzione, i Colleghi di tutte le Università per la loro partecipazione alle diverse iniziative create intorno alla rivista, il CUEIM che con il suo sostegno ha reso possibile la pubblicazione della rivista e la Redazione tutta senza la quale questa lunga strada non sarebbe certo stata percorsa.

Insieme siamo riusciti ad arrivare sin qui, insieme sapremo camminare in sintonia di pensiero e azioni verso ulteriori e sempre più sfidanti traguardi consapevoli che l'“unica costante oggi è il cambiamento" (Giovanni Panati).

Claudio Baccarani

Gaetano M. Golinelli
Claudio Baccarani Gaetano M. Golinelli Eccoci al numero 100 di Sinergie 